\title{
Gerencia de materiales en la industria azucarera venezolana
}

\author{
Pico Pico, Gonzalo * \\ Galván Rico, Luis Enrique **
}

\section{Resumen}

Este trabajo aborda el problema de la gerencia de materiales en Venezuela, especificamente en la industria azucarera. Se pretende analizar aspectos organizacionales y de recursos humanos, los cuales condicionan el grado de eficiencia que es deseable alcanzar en la función de administrar los materiales. Como resultado del estudio se logró determinar que la estructura de la organización de materiales es arcaica, haciéndose necesaria su descentralización. Por otra parte, el personal responsable no tiene, en general, las capacidades requeridas para trabajos de este tipo.

Palabras clave: Gerencia de materiales, industria azucarera, inventarios.

\section{Recibido: 98-11-03 . Aceptado: 99-04-12}

Los autores son Profesores de la Universidad Simón Bolivar, Sede del Litoral.

* Ing. Industrial, Especialista en Administración, Mención Gerencia General.

** Economista, Msc. en Gerencia Empresarial. Dirección: Valle de Camurí Grande, Parroquia Naiguatá, Territorio Vargas. Tlf: 031-901131/901159. Tele-Fax: 02-9532876. Apartado Aéreo 50923, Sabana Grande, Caracas. 


\section{Management of Materials in the Venezuelan Sugar Industry}

\section{Abstract}

This work addresses the problem of the management of materials in Venezuela, specifically in the sugar industry. It analyzes the organizational and human resource aspects which condition the degree of efficiency desired in the administration of materials. As a result of the study, it was determined that the structure of the organization of materials is archaic, and that its decentralization is needed. Furthermore, the personnel responsible for it do not have, in general, the capacities required for this type of work.

Key words: Management of materials, sugar industry, inventories.

\section{Introducción}

La industria azucarera constituye una de las actividades agroindustriales más importantes de Venezuela, tanto por su contribución al desarrollo agricola e industrial como por su capacidad para generar empleo. Según el Directorio Industrial Azucarero, la agroindustria azucarera es fuente directa de trabajo para más de $\mathbf{3 5 . 0 0 0}$ personas, a las cuales se suman 150.000 que trabajan de manera indirecta. En el campo se estima la ocupación en época de zatra, para labores de cultivo, corte, alza y transporte de la caña de azúcar, en 30.000 personas. Por otra parte, existen unas 4.000 fincas cuya extensión aproximada es de 100.000 hectáreas, en las cuales se siembran y cultivan 6.500 .000 toneladas de caña año tras año, que generan unas 430.000 toneladas de azúcar.

Además, la industria azucarera contribuye mediante la producción de azúcar, melaza y bagazo, al desarrollo de otras empresas e industrias nacionales en rubros tales como la fabricación de alcohol, tableros aglomerados, alimentos para ganadería, levadura, papel, bebidas, golosinas, conservas, congelados y muchos otros productos. Según la Fundación Venezolana para la Investigación y la Productividad en el Sector Azucarero, FUNDAZUCAR (1994), los inventarios de materiales representaban en 1994 un valor promedio anual aproximado del $12 \%$ del total de los activos fijos. El valor de estos inventarios asciende a una cifra considerable; por ejemplo, la importación anual de materiales para la industria azucarera está hoy en dia por el orden de los 3.5 millardos de bolivares.

De lo anterior se desprende la importancia que tiene para dicha industria y por consiguiente para la economía nacional, un diagnóstico de la gestión de materiales, más aún si se tiene en cuenta que hasta el presente no se ha realizado un 
estudio que analice de manera sistemática la gerencia de materiales en tan importante sector de la industria nacional.

En la adquisición y almacenamiento de materiales se emplean grandes sumas de dinero, las cuales se van incrementando a medida que las empresas crecen en magnitud y complejidad; debido a ello, la racionalización de los recursos invertidos, la disminución de los costos y de los riesgos de agotamiento de los materiales, son aspectos importantes a tener en cuenta, ya que afectan el nivel de productividad que se puede alcanzar en las empresas (Diaz, 1991). Pero en esta búsqueda de productividad es necesario tener en cuenta otros aspectos no menos importantes de la administración de materiales, como son la organización y los recursos humanos, los cuales condicionan de manera permanente el grado de eficiencia que se pretende alcanzar. En el análisis de la idoneidad de estos aspectos, se centra el presente estudio.

La población estuvo constituida por los centrales azucareros tanto privados como aquellos que son propiedad del Estado Venezolano. Existen en total 19 centrales azucareros, de los cuales 12 son públicos y los 7 restantes, privados, a los cuales se aplicó un cuestionario cuya validación se hizo a través del juicio de expertos, pertenecientes a las áreas de administración de materiales y de gerencia. Además de la aplicación del cuestionario, se realizaron entrevistas con personas responsables del área de materiales, con el fin de complementar la información.

El instrumento definitivo se aplicó en cada uno de los centrales azucareros que conforman la población objeto de estudio. En el análisis e interpretación de los resultados derivados de la aplicación del instrumento, se utilizó como nivel de medición la escala nominal o de clasificación y se determinó la frecuencia como elvalor estadístico apropiado para dicho análisis; este valor estadístico se obtuvo por medio de la aplicación del paquete estadístico computarizado SPSS (Statistical Package for the Social Sciences), versión 5.0 .

\section{Análisis de la organización de materiales}

El tipo de materiales a los cuales se refiere esta investigación, son todos aquellos que se requieren para el rendimiento óptimo de las operaciones de los centrales azucareros, tomando en consideración los factores de demanda, costos y tiempo, asi como el costo de mantener un cierto nivel de existencias que permitan lograr un balance económico y reducir los riesgos de agotamiento. De estos materiales se excluyen los conceptos de materia prima y productos terminados.

Los materiales se clasifican en tres grupos $(A, B, C)$ bajo la aplicación del Principio de Pareto, dividiendo los inventarios para efectos de control en tres tipos de artículos: al grupo A pertenecen los pocos materiales que tienen un alto valor en dinero; el grupo B está compuesto por aquellos materiales que representan un valor moderado, y el grupo $\mathrm{C}$, lo constituyen un gran número de materiales que tienen un valor reducido. Esta clasificación tiene como objetivo enfocar la mayor parte de los recursos y del esfuerzo en el control de los materiales criticos y no en los muchos triviales (Render y Heizer, 1996). 
En la adquisición y almacenamiento de estos materiales se emplean grandes sumas de dinero, las cuales se van incrementando a medida que las empresas crecen en magnitud y complejidad. Debido a ello, la racionalización de los recursos invertidos, la disminución de los costos y de los riesgos de agotamiento de los materiales, son aspectos importantes a tener en cuenta, ya que afectan el nivel de productividad que se puede alcanzar en las empresas (Díaz, 1991). Pero en la búsqueda de productividad es necesario considerar otros aspectos no menos importantes de la administración de materiales, como son los aspectos organizacionales y los relativos a los recursos humanos, los cuales condicionan de manera permanente el grado de eficiencia que se pretende alcanzar.

En cuanto a los aspectos organizacionales, manifiesta Díaz que al plantearse estrategias, las empresas deben asegurarse que sus estructuras productivas e infraestructuras operativas estén muy bien sincronizadas; el no hacerlo ocasiona plantas industriales funcionando al $50 \%$ de su capacidad y procedimientos administrativos que, aunque estén bien diseñados, únicamente funcionan el primer mes. Además, de los elementos que componen esta infraestructura, como la estructura organizativa y los sistemas de información, entre otros, el mantenimiento ha sido particularmente descuidado.

El análisis de la información obtenida permitió determinar que en la totalidad de los centrales azucareros encuestados, la organización de materiales está conformada por la Unidad de Compras y el Almacén de Materiales; ambas unida- des están centralizadas y dependen de la Gerencia de Administración. Ya desde 1973 Mosquera consideraba arcaico este tipo de esquema, porque depende del área administrativa y acata decisiones ya tomadas por el área técnica.

Por otra parte, de acuerdo con Berman (1979), la gestión de materiales presenta características típicas de los casos en los cuales es conveniente su descentralización, a saber: problemas de rutina a corto plazo, problemas de gestión general y problemas de políticas. En razón de lo anterior, puede afirmarse que la función de gestión de materiales en los centrales azucareros no tiene el grado de centralización/descentralización adecuado y la ubicación de la unidad de gestión de materiales no corresponde al nivel jerárquico adecuado para posibilitar la toma de decisiones oportunas y correctas.

En cuanto a la codificación de los materiales ubicados en el almacén, en la mayoría de los centrales consultados se utilizan dos tipos de códigos diferentes: uno para la administración de los materiales y otro para efectos contables. El primer tipo de código, a pesar de ser diferente en cada central, describe apropiadamente los materiales. Por ejemplo, en el caso del Central Río Turbio, los dígitos del código identifican el tipo de material, el número de la tarjeta y un número correlativo.

En todos los casos, el personal del almacén de materiales no dispone de un instructivo referente al manejo de los códigos de los materiales y, por lo tanto, dependen de la memoria del personal que los maneja. El código que está bastante generalizado entre los centrales azucareros es el código de ubicación, el cual identifica la localización dentro del almacén. 
Este código consta de una letra y tres dígitos, que identifican el número de la ubicación, el número del estante y la letra de la sección o pasillo.

El control de los materiales se lleva por computadora, con la cual se procesan los documentos que respaldan los movimientos de los materiales. Los catálogos de los fabricantes y proveedores, además de sus direcciones y teléfonos, se mantienen organizados en el departamento de compras.

Por otra parte, según lo manifestado por Armstrong (1991), lo ideal es que, como resultado de un análisis de actividades, se señale a cada quien lo que se está haciendo, lo que no se está haciendo y lo que debe hacerse; sin embargo, ninguno de los centrales azucareros posee un manual de organización ni descripciones de los cargos correspondientes al área de materiales. Las entrevistas realizadas permitieron establecer que el personal conoce suficientemente los procedimientos, funciones, deberes y responsabilidades propios de los cargos que desempeñan, como producto de su experiencia.

\section{Supervisión, capacitación y carga laboral}

Los recursos humanos constituyen la parte más importante de cualquier organización $y$, de acuerdo con Narasimhan, McLeavey y Billington (1996), nada se podrá hacer bien a menos que el personal se capacite, se prepare y esté motivado para trabajar con el propósito de alcanzar el éxito. La falta de capacitación y motivación resultan en un personal no idóneo para desempeñarse en el área de administración de materiales.
Por supuesto, la capacitación también involucra a los gerentes en el área de materiales; aquellas personas que creen haber completado su profesionalización y están convencidas de saber todo lo que hay que saber al respecto, pronto dejarán de serverdaderos profesionales. Menciona Messner (1986), a manera de ejempio, que aunque se haya obtenido el título de Gerente de Compras, se debe continuar el proceso de aprendizaje permanente $y$, como gerente, debe mantener a su gente involucrada en dicho proceso.

Por otra parte, opina Mosquera (1994) que nadie en el área de materiales deberá estar comprometido e involucrado de manera directa o indirecta, mediante lazos familiares, con ninguna organización que esté haciendo negocios con la empresa; esto, con el fin de evitar conflictos de intereses.

El aspecto más relevante estudiado fue la distribución de la carga de trabajo. Al respecto, de acuerdo con los resultados obtenidos, en el $61 \%$ de los centrales azucareros hay un promedio de 65 artículos movilizados por persona y por mes; mientras en el $28 \%$ el promedio es de 75 artículos y sólo en el $11 \%$ de los centrales azucareros el promedio es de 86.6 artículos. Las cifras anteriores reflejan una carga de trabajo aceptable, si se considera que, según la experiencia de los involucrados, el valor normal está entre 50 y 100 artículos a ser movilizados por persona y por mes. Sin embargo, haciendo la conversión a días, obtenemos valores que oscilan entre $21 / 2$ y $31 / 2$ artículos movilizados diariamente por cada trabajador, lo cual resulta una carga baja de trabajo. Estos movimientos de materiales corresponden a requisiciones, colocacio- 
nes de órdenes de compra, recepción y devoluciones.

En la totalidad de los centrales azucareros, ninguno de los supervisores del área de materiales tiene bajo su mando más de 5 personas. De lo anterior se desprende que la distribución de actividades de supervisión entre el personal de la unidad de administración de materiales, permite cumplir estas actividades apropiadamente, pues, según el principio de ámbito de control descrito por Schultz (1985), es una cantidad adecuada.

En la gran mayoria de los centrales azucareros, las personas que ocupan los diferentes cargos de responsabilidad dentro de la unidad de administración de materiales no son profesionales universitarios graduados en el área de materiales, sino personal sin título universitario o que, aún teniéndolo, no lo poseen en el área de materiales. En ambos casos, ha sido a través de la experiencia como han adquirido conocimientos y habilidades propios de los cargos que ocupan. Por otra parte, sólo en casos aislados, algunas personas que trabajan en el área de materiales han realizado cursos relacionados con las funciones que desempeñan.

Por lo anteriormente expuesto, en general, el personal que trabaja en el área de materiales no tiene el perfil requerido, por to cual puede afirmarse que el personal encargado de la administración de los materiales en los centrales azucareros en Venezuela no es, en la mayoría de los casos, idóneo.

\section{Conclusiones}

La estructura típica de la organización de materiales es arcaica por depen- der del área administrativa y acatar decisiones que toma el área técnica. Se recomienda entonces un cambio estructural que elimine su dependencia del área administrativa y pase a ser soporte técnico de otras áreas, quedando al mismo nivel de producción y mantenimiento.

La gestión de materiales presenta características propias de los casos en que se hace necesaria la descentralización. Se propone descentralizar la organización de materiales, asignando al responsable de cada área operativa la administración de aquellos materiales que corresponden al funcionamiento de su unidad. Además, la tramitación de las compras como tal, se debe centralizar en una unidad administrativa.

En general, el personal encargado de la administración de materiales no tiene las condiciones de idoneidad requeridas para desempeñarse en trabajos de este tipo. Considerando la dinámica actual existente en el área de materiales, así como el continuo surgimiento de técnicas que permiten optimizar la gestión en esa área, se hace necesario para los centrales azucareros contar con personal que tenga niveles de formación suficientes para estar en capacidad de asimilar dichas técnicas. El nivel que corresponde al perfil de un administrador en el área de materiales es el de un pro. fesional universitario, preferiblemente ingeniero industrial. En consecuencia, se recomienda que la Gerencia de recursos humanos en la industria azucarera se oriente al reclutamiento y selección de personal con las características mencionadas y a su adiestramiento y desarrollo, con el fin de mantenerlo permanentemente actualizado. 


\section{Bibliografía citada}

Armstrong, Michael (1991). Gerencia de Recursos Humanos, Legis Editores, p. 43 , Bogotá..

Berman, Josué (1979). Centralización y Descentralización. Diecisiete Preguntas y Algunas Respuestas, Revista Administración de Empresas, Año IX, Núm. 108, Buenos Aires.

Diaz, Angel (1991). Gestión de Inventario en Mantenimiento, Ediciones IESA, p. 7 y 21-22, Caracas.

Hernández, Roberto; Fernández, Carlos; Baptista, Pllar (1991). Metodología de la Investigación, Editorial McGraw-Hill, p.58-64, Bogotá.

Messner, William (1986). La Gerencia de Compras, Editorial Norma, p. 368, Colombia.
Mosquera, Genaro (1973). Sistemas de Control de Gestión, Facultad de Ciencias Económicas y Sociales, UCV, Caracas.

Mosquera, Genaro (1994). Gerencia de Logística Industrial, Fundación Editorial Universitaria de Venezuela, p. 187, Caracas.

Naghi, Mohammad (1990). Metodología de la Investigación, Cuarta Edición, Editorial Limusa, p. 167-168, México.

Narasimhan, Sim; McLeavey, Dennis; Billington. Peter (1996). Planeación de la Producción y Control de Inventa-

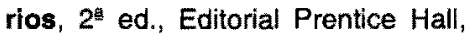
p. 10 y 350 , México.

Schultz, David (1985). Psicología Industrial, Nueva Editorial Interamericana, p. 282, México. 